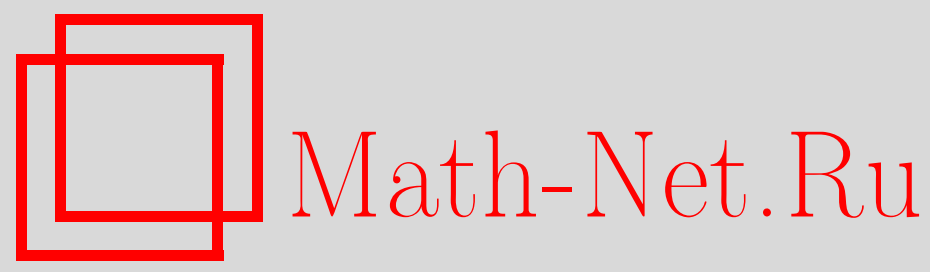

В. Н. Княгина, В. С. Монахов, Конечные группы с нильпотентными и холловыми подгруппами, Дискрет. матем., 2013, том 25, выпуск 1, 137-143

DOI: https://doi.org/10.4213/dm1226

Использование Общероссийского математического портала Math-Net.Ru подразумевает, что вы прочитали и согласны с пользовательским соглашением http://www . mathnet.ru/rus/agreement

Параметры загрузки:

IP : 54.198 .187 .58

26 апреля 2023 г., 18:06:28 


\title{
Конечные группы с нильпотентными и холловыми подгруппами
}

\author{
() 2013 г. В. Н. Княгина, В. С. Монахов
}

\begin{abstract}
Устанавливается строение конечной группы, у которой каждая подгруппа либо нильпотентна, либо холлова.
\end{abstract}

Конечную ненильпотентную группу, все собственные подгруппы которой нильпотентны, называют группой Шмидта. Впервые эти группы рассматривались в работе О. Ю. Шмидта [1]. Группам Шмидта посвящен отдельный параграф монографии Хупперта [2] (см. §III.5). Обзор результатов о группах Шмидта и перспективы их приложений в теории групп по состоянию на 2001 г. приведен в [3].

Если в конечной группе все подгруппы холловы, то, очевидно, ее порядок свободен от квадратов, то есть не делится на $p^{2}$ для любого простого $p$. По теореме IV.2.11 в [2], такая группа содержит нормальную циклическую холлову подгруппу, фактор-группа по которой циклическая.

В настоящей заметке изучаются свойства конечной ненильпотентной группы, в которой каждая подгруппа либо холлова, либо нильпотентна. Доказывается следующая теорема.

Теорема 1. Пусть $G$ - конечная ненильпотентная группа, в которой каждая подгруппа либо холлова, либо нильпотентна. Тогда справедливы следующие утверждения:

(1) если силовская р-подгруппа $P$ ненормальна в $G$, то $P$ цчиклическая и каждая максимальная подгруппа из $P$ содержится в иентре $G$;

(2) если силовская р-подгруппа $P$ нормальна в $G$, то либо $P$-минимальная нормальная в $G$ подгруппа, либо $P$ неабелева,

$$
Z(P)=P^{\prime}=\Phi(P),
$$

и $P / \Phi(P)-$ минимальная нормальная в $G / \Phi(P)$ подгруппа;

(3) если $P_{1}-$ нормальная р-подгруппа, отличная от силовской, то $P_{1}$ содержится 6 иентре $G$;

(4) фактор-группа $G / Z(G)$ содержит нормальную абелеву холлову подгруппу $A / Z(G)$, y которой каждая силовская подгруппа является минимальной нормальной в $G / Z(G)$ подгруппой, а $G / A-$ цииклическая группа порядка, свободного от квадратов. 
Следствие 1. Пусть $G$ - конечная ненильпотентная группа, в которой каждая подгруппа либо холлова, либо нильпотентна. Тогда $G$ содержит нильпотентную холлову подгруппу, фактор-группа по которой ичикическая. В частности, производная длина $G$ не выме 3.

Рассматриваются только конечные группы. Используемые терминология и обозначения соответствует $[2,4]$. Запись $G=[A] B$ означает полупрямое произведение с нормальной подгруппой $A$, а $\pi(G)$ - множество всех простых делителей порядка группы $G$. Знакопеременная и симметрическая группы степени $n$ обозначаются через $A_{n}$ и $S_{n}$, а $E_{p^{n}}$ и $Z_{m}$ - элементарная абелева группа порядка $p^{n}$ и циклическая группа порядка $m$ соответственно. Центр, коммутант, подгруппы Фраттини, Фиттинга и силовская $p$-подгруппа группы $G$ обозначаются через $Z(G), G^{\prime}, \Phi(G), F(G)$ и $G_{p}$ соответственно. Дисперсивной называют группу, обладающую нормальным рядом, факторы которого изоморфны силовским подгруппам группы.

Пусть $\mathfrak{\Re}$ - класс всех нильпотентных групп. Через $H(\mathfrak{N})$ обозначим класс всех групп, у которых каждая подгруппа либо холлова, либо нильпотентна. Ясно, что все нильпотентные группы, все группы Шмидта и все группы, порядки которых свободны от квадратов, принадлежат $H(\mathfrak{N})$. Бипримарные ненильпотентные группы из класса $H(\Re)$ являются группами Шмидта. Ниже приведены другие примеры групп из этого класса.

Пример 1. Пусть $G=A \times S,(|A|,|S|)=1,|A|$ свободен от квадратов, $A$ нильпотентна, $S-$ группа Шмидта. Пусть $\pi=\pi(A), \sigma=\pi(S)$. Тогда $A-\pi$-холлова, $S-\sigma$-холлова подгруппы в $G$. Пусть $K-$ произвольная подгруппа из $G$. Тогда ее $\pi$-холлова подгруппа $K_{\pi}$ содержится в $A$ и $K_{\pi}=A \cap K, K_{\sigma} \subseteq S$, и $K_{\sigma}=K \cap S$, поэтому $K=K_{\pi} \times K_{\sigma}$. Если $K_{\sigma} \neq S$, то $K_{\sigma}$ нильпотентна и $K$ нильпотентна. Пусть $K_{\sigma}=S$, то есть $K=(K \cap A) \times S$. Так как $|A|$ свободен от квадратов, то все подгруппы в $A$ холловы и $K-$ холлова в $G$. Итак, $G \in H(\Re)$.

Пример 2. Пусть $P$ - экстраспециальная группа порядка $409^{3}$. Ясно, что

$$
\Phi(P)=Z(P)=P^{\prime}
$$

- подгруппа простого порядка 409 и $P / \Phi(P)$ - элементарная абелева группа порядка $409^{2}$. Группой автоморфизмов $P / \Phi(P)$ является группа $G L(2,409)$, в которой по теореме II.7.3 из [6] существует циклическая подгруппа $Z_{210}$ порядка 5 4 41. Группа $Z_{210}$ действует неприводимо на $P / \Phi(P)$. Поэтому существует группа $T=[P] Z_{210}$, у которой подгруппа $\Phi(P)$ совпадает с центром $T$. В группе $T$ максимальные подгруппы исчерпываются следующими подгруппами: $[P] Z_{5}$ - группа Шмидта; $[P] Z_{41}$ - группа Шмидта; $\Phi(P) \times Z_{210}-$ нильпотентная подгруппа. Следовательно, $T$ является трипримарной группой, у которой каждая подгруппа либо нильпотентна, либо группа Шмидта.

Пример 3. Пусть

$$
G=\left[\left(E_{4} \times E_{25} \times E_{7} \times E_{121} \times E_{169} \times \cdots\right)\right] Z_{3},
$$

где $\left[E_{4}\right] Z_{3},\left[E_{25}\right] Z_{3},\left[E_{7}\right] Z_{3},\left[E_{121}\right] Z_{3},\left[E_{169}\right] Z_{3}, \ldots-$ - группы Шмидта, в которых все собственные подгруппы примарны. Пусть $K-$ произвольная подгруппа из $G$. Если 3 не делит порядок $K$, то $K$ нильпотентна. Пусть 3 делит $|K|$. Так как $G p$-замкнута для любого $p \neq 3$, то $K p$-замкнута и существует подгруппа $\left[K_{p}\right] Z_{3}$. По теореме Холла, $\left[K_{p}\right] Z_{3} \subseteq\left[G_{p}\right] Z_{3}$. Но в последней группе все собственные подгруппы примарны. Поэтому либо $\left[K_{p}\right] Z_{3}=\left[G_{p}\right] Z_{3}-$ холлова, либо $K_{p}=1$. Так как $p-$ произвольное $\neq 3$, то $K-$ холлова в $G$ и $G \in H(\Re)$. 
Лемма 1. Если $G \in H(\Re)$, то каждая подгруппа из $G$ и каждая фактор-группа группь $G$ принадлежат $H(\mathfrak{N )}$.

Доказательство. Пусть $U \leqslant V \leqslant G \in H(\Re)$. Предположим, что $U$ ненильпотентна. Тогда $U-$ холлова подгруппа в $G$ поскольку $G \in H(\mathfrak{N})$. Ясно, что $U-$ холлова подгруппа в $V$. Значит, $V \in H(\mathfrak{⿰})$.

Пусть $N-$ нормальная подгруппа группы $G, G \in H(\mathfrak{N})$ и $X / N \leqslant G / N$. Предположим, что $X / N$ ненильпотентна. Тогда $X$ ненильпотентна, а значит $X-$ холлова подгруппа в $G$. Но теперь $X / N-$ холлова подгруппа в $G / N$ и $G / N \in H(\mathfrak{F})$.

Лемма 2 ([1, 3]). Пусть $S$ - группа Шмидта. Тогда справедливы следующие утверждения:

(1) $S=[P]\langle y\rangle$, где $P-$ нормальная силовская р-подгруппа, $\langle y\rangle-$ ненормальная ичиклическая силовская q-подгруппа, p и q-различные простые числа, $y^{q} \in Z(S)$;

(2) $\left|P / P^{\prime}\right|=p^{m}$, где $m-$ показатель числа $p$ по модулю $q$;

(3) если $P$ абелева, то $P$ - элементарная абелева порядка $p^{m}$ и $P-$ минимальная нормальная в $S$ подгруппа;

(4) если $P$ неабелева, то $Z(P)=P^{\prime}=\Phi(P)$ u $|P / Z(P)|=p^{m}$;

(5) если $P_{1}-$ неединичная нормальная р-подгруппа группы $S$, отличная от $P$, то $P$ неабелева и $P_{1} \subseteq Z(P)$;

(6) $Z(S)=\Phi(S)=\Phi(P) \times\left\langle y^{q}\right\rangle ; S^{\prime}=P, P^{\prime}=\left(S^{\prime}\right)^{\prime}=\Phi(P)$;

(7) если $N-$ собственная нормальная подгруппа из $S$, то подгруппа $\langle y\rangle$ не содержится в $N$ и либо $P$ содержится в $N$, либо $N$ содержится в $\Phi(S)$.

Напомним, что $p d$-подгруппа - это подгруппа, порядок которой делится на $p$. Группа с нормальной силовской $p$-подгруппой называется $p$-замкнутой, а группа с нормальной $p^{\prime}$-холловой подгруппой $-p$-нильпотентной. Группа, которая одновременно $p$-замкнута и $p$-нильпотентна, называется $p$-разложимой.

Лемма 3. (1) Если группа не р-нильпотентна, то в ней существует р-замкнутая pd-подгруппа Шмидта.

(2) Если группа не 2-замкнута, то в ней существует 2-нильпотентная подгруппа Шмидта четного порядка.

(3) Если р-разрешимая группа не р-замкнута, то в ней существует р-нильпотентная pd-подгруппа Шмидта.

Доказательство. Первое утверждение вытекает из теоремы Фробениуса (см. [2], теорема IV.5.4).

Докажем второе утверждение. В [5] имеется доказательство, основанное на теореме Судзуки о простых группах с независимыми силовскими 2-подгруппами. Приведем другое доказательство. По индукции, все собственные в $G$ подгруппы 2-замкнуты и группа $G$ не бипримарна по п. 1. Если группа $G$ разрешима, то все бипримарные холловы подгруппы 2-замкнуты, поэтому и группа $G$ 2-замкнута. Приходим к противоречию. Значит, $G$ неразрешима. Ясно, что фактор-группа $G / \Phi(G)$ - простая. Пусть $X-$ класс сопряженных инволюций в $G / \Phi(G)$. По теореме IX.7.8 в [6], существуют две инволюции $x$ и $y \in X$ 
такие, что их порождение $\langle x, y\rangle$ не является 2-группой. Хорошо известно, что $\langle x, y\rangle-$ диэдральная группа порядка $2|x y|$ (см. [4], теорема 2.49), которая не будет 2-замкнутой. Опять приходим к противоречию.

Докажем третье утверждение. По теореме 5.3 .13 в [7], группа $G$ является $D_{\{p, q\}}$ группой для любого $q \in \pi(G)$. Пусть $G$ не $p$-замкнута. Тогда существует $q \in \pi(G)$ такое, что $\{p, q\}$-холлова подгруппа $H$ из группы $G$ не $p$-замкнута. Ясно, что $H$ не $q$-нильпотентна. По п. 1 в $H$ существует $p$-нильпотентная $p d$-подгруппа Шмидта.

Для любого нечетного $p$ аналог утверждения 2 леммы 3 неверен. При $p=3$ контрпримером служит простая группа $S L\left(2,2^{n}\right)$ при любом нечетном $n$, а при $p \geqslant 5$ - группа $\operatorname{PSL}(2, p)$.

Лемма 4. Если $G \in H(\mathfrak{⿰})$, то $G$ дисперсивна.

Доказательство. Вначале докажем, что если $G \in H(\Re)$ и $p-$ наименьшее в $\pi(G)$, то либо $G$-замкнута, либо $p$-нильпотентна. Предположим, что $p=2$. Если группа $G$ не 2-замкнута, то, по утверждению 2 леммы 3 , в $G$ существует 2-нильпотентная подгруппа Шмидта $S$ четного порядка. Силовская 2-подгруппа в $S$ циклическая. Так как $S$ ненильпотентна и $G \in H(\mathfrak{N})$, то $S$ холлова, поэтому силовская 2-подгруппа в $G$ циклическая. Теперь $G$ 2-нильпотентна по теореме IV.2.8 в [2]. Пусть $p>2$. Тогда $G$ разрешима. Если группа $G$ не $p$-замкнута, то, по утверждению 3 леммы 3 , в $G$ существует $p$-нильпотентная $p d$-подгруппа Шмидта $T$. Силовская $p$-подгруппа $P$ в $T$ циклическая. Так как $T$ ненильпотентна и $G \in H(\Re)$, то $T$ холлова и $P$ - силовская $p$-подгруппа группы $G$. Теперь $G$ p-нильпотентна по теореме IV.2.8 в [2].

Итак, если $G \in H(\mathfrak{⿰})$ и $p$ - наименьшее в $\pi(G)$, то либо $G$-замкнута, либо $p$-нильпотентна. Теперь для доказательства дисперсивности воспользуемся индукцией по порядку группы. Пусть $p$ - наименьшее в $\pi(G)$. Если силовская $p$-подгруппа $P$ нормальна в $G$, то $G / P \in H(\mathfrak{⿰})$ по лемме 1 , а по индукции $G / P$ дисперсивна. Поэтому $G$ дисперсивна. Если $G p$-нильпотентна, то в $G$ имеется нормальная подгруппа $K$ и $G / K$ изоморфна силовской $p$-подгруппе группы $G$. По лемме $1, K \in H(\mathfrak{N})$, а по индукции подгруппа $K$ дисперсивна. Поэтому $G$ дисперсивна. Лемма доказана.

Лемма 5. Пусть $G \in H(\mathfrak{N}) u p, q \in \pi(G), p \neq q$. Тогда $\{p, q\}$-холлова подгруппа в $G$ либо нильпотентна, либо группа Шмидта.

Доказательство. Ввиду леммы 4, группа $G$ разрешима, поэтому в ней существует $\{p, q\}$ холлова подгруппа $K$. Каждая собственная подгруппа в $K$ либо силовская, либо нильпотентная $\{p, q\}$-подгруппа ввиду условия $G \in H(\mathfrak{N})$. Значит, $K$ либо нильпотентна, либо группа Шмидта.

Лемма 6. Пусть $n \geqslant 2$ - натуральное, $p-$ простое $и \pi-$ конечное множество простых чисел q таких, что q делит $p^{n}-1$ и q не делит $p^{n_{1}}-1$ для всех $1 \leqslant n_{1}<n$. Тогда группа $G L(n, p)$ содержит циклическую $\pi$-холлову подгруппу.

Доказательство. Группа $G=G L(n, p)$ имеет, очевидно, порядок, равный $p^{n(n-1) / 2} \times$ $\left(p^{n}-1\right)\left(p^{n-1}-1\right) \cdots\left(p^{2}-1\right)(p-1)$. По теореме II.7.3 в [2], группа $G$ содержит циклическую подгруппу $T$ порядка $p^{n}-1$. Ее $\pi$-холлова подгруппа $T_{\pi}$ будет, очевидно, $\pi$-холловой подгруппой группы $G$ ввиду того, что $q$ не делит $p^{n_{1}}-1$ для всех $q \in \pi$ и всех $1 \leqslant n_{1}<n$. 
Доказательство теоремы 1. Докажем первое утверждение. Пусть $G \in H(\mathfrak{⿰})$ и $p \in \pi(G)$. Предположим, что силовская $p$-подгруппа $P$ ненормальна в $G$. По лемме 4 , группа $G$ разрешима, поэтому в $G$ существует $\{p, q\}$-холлова подгруппа для любого $q \in \pi(G) \backslash\{p\}$, см. [7], теорема 5.3.13. Подгруппа $P$ ненормальна в $G$, значит существует не $p$-замкнутая $\{p, q\}$-холлова подгруппа $K$ для некоторого $q \in \pi(G) \backslash\{p\}$. В $K$ имеется, по лемме 3 , $q$-замкнутая подгруппа Шмидта, которая совпадает с $K$ ввиду условия $G \in H(\mathfrak{N})$. По свойствам групп Шмидта (лемма 2 , п. 1) силовская $p$-подгруппа в $K$ циклическая. Так как $K$ - холлова подгруппа в $G$, то силовская $p$-подгруппа из $K$ будет силовской в $G$. Итак $P$ - циклическая.

Пусть $P_{1}$ - максимальная подгруппа из $P$. Если $P_{1}=1$, то $P_{1} \subseteq Z(G)$. Пусть $P_{1} \neq 1$. Для любого простого $q \in \pi(G) \backslash\{p\}$ в $G$ существует $\{p, q\}$-холлова подгруппа $P Q$, где $Q$ - некоторая силовская $q$-подгруппа из $G$. Если $P Q$ нильпотентна, то $Q \subseteq C_{G}\left(P_{1}\right)$. Если $P Q$ ненильпотентна, то $P Q$ является группой Шмидта по лемме 5. Если $P Q p$-замкнута, то $P$ имеет простой порядок (лемма 2, п. 3), получаем противоречие. Значит, $P Q q$ замкнута и $P_{1} \subseteq Z(P Q)$ (лемма 2 , п. 1), то есть $Q \subseteq C_{G}\left(P_{1}\right)$. Итак, $C_{G}\left(P_{1}\right)$ содержит некоторую силовскую $q$-подгруппу для каждого $q \in \pi(G) \backslash\{p\}$. Так как $P \subseteq C_{G}\left(P_{1}\right)$, то $C_{G}\left(P_{1}\right)=G$ и $P_{1} \subseteq Z(G)$.

Докажем второе утверждение. Пусть силовская $p$-подгруппа $P$ нормальна в $G$ и $P$ не является минимальной нормальной в $G$ подгруппой, в частности, $|P|>p$. По теореме Шура-Цассенхауза, в $G$ существует $p^{\prime}$-холлова подгруппа $H$. Если $G=P \times H$, то $H$ ненильпотентна и в $H$ существует подгруппа Шмидта $S$. Теперь для подгруппы $P_{1}$ простого порядка из $P$ подгруппа $P_{1} \times H$ будет ненильпотентной и нехолловой, получаем противоречие. Значит, допущение $G=P \times H$ неверно, и в $H$ существует силовская подгруппа $Q$, такая, что $[P] Q$ ненильпотентна. По лемме $5,[P] Q-$ подгруппа Шмидта. Согласно предположению, $P$ не является минимальной нормальной в $G$ подгруппой, поэтому $P$ не будет минимальной нормальной подгруппой в $[P] Q$. Из свойств групп Шмидта (лемма 2, п. 3) следует, что $P$ неабелева и

$$
Z(P)=P^{\prime}=\Phi(P)
$$

Так как $[P / Z(P)](Q Z(P) / Z(P)$ - подгруппа Шмидта с минимальной нормальной подгруппой $P / Z(P)$, то $P / Z(P)$ - минимальная нормальная в $G / Z(P)$ подгруппа.

Докажем третье утверждение. Предположим, что $P_{1}$ не содержится в $Z(G)$. Тогда $\left|P_{1}\right| \geqslant p,\left|G_{p}\right| \geqslant p^{2}$ и силовская $p$-подгруппа $G_{p}$ нормальна по уже доказанному утверждению 1 теоремы. Пусть $G_{q}$ - произвольная силовская $q$-подгруппа группы $G$, $q \in \pi(G) \backslash\{p\}$. По лемме 5, подгруппа $G_{p} G_{q}$ либо нильпотентна, либо группа Шмидта. Предположим, что $G_{p} G_{q}$ нильпотентна для всех $q \in \pi(G) \backslash\{p\}$. Тогда $G=G_{p} \times G_{p^{\prime}}$. Так как $G$ не нильпотентна, то подгруппа $G_{p^{\prime}}$ не нильпотентна, а значит в $G_{p^{\prime}}$ существует подгруппа Шмидта $S$. Но теперь подгруппа $P_{1} \times S$ будет не холловой в $G$ и не нильпотентной, получаем противоречие. Значит, допущение неверно, и существует $r \in \pi(G) \backslash\{p\}$ такое, что $G_{p} G_{r}$ не нильпотентна. Но теперь $G_{p} G_{r}-p$-замкнутая группа Шмидта с нормальной $p$-подгруппой $P_{1}$. По свойствам подгрупп Шмидта (лемма 2, п. 5), $P_{1} \subseteq Z\left(G_{p} G_{r}\right)$. Итак, $P_{1} \subseteq Z\left(G_{p}\right)$ и $G_{r} \subseteq C_{G}\left(P_{1}\right)$ для всех $r \in \pi(G) \backslash\{p\}$, у которых $G_{p} G_{r}$ не нильпотентна. Если $G_{p} G_{r}$ нильпотентна, то $G_{q} \subseteq C_{G}\left(P_{1}\right)$. Значит, $P_{1} \subseteq Z(G)$.

Докажем четвертое утверждение. Пусть $\mathfrak{A}, \mathfrak{N}$ и $\mathfrak{5}$ - классы всех абелевых, нильпотентных и всех конечных групп соответственно, а

$$
\mathfrak{N} \mathfrak{U}=\left\{G \in \mathfrak{F} \mid G^{\mathfrak{A}} \in \mathfrak{N}\right\}
$$


- произведение классов $\mathfrak{\Re}$ и $\mathfrak{U}$ (см. [4], с. 167). Здесь $G^{\mathfrak{U}}-\mathfrak{U}$-корадикал группы, то есть наименьшая нормальная подгруппа, фактор-группа по которой принадлежит $\mathfrak{A}$. Ясно, что $G^{\mathfrak{Q}}=G^{\prime}$ - коммутант группы $G$, поэтому класс $\mathfrak{N} \mathfrak{U}$ состоит из всех групп с нильпотентным коммутантом, и этот класс является насыщенной формацией. С помощью индукции по порядку группы покажем, что $H(\mathfrak{N}) \subseteq \mathfrak{N} \mathfrak{A}$. Допустим противное, и пусть $G-$ группа наименьшего порядка, принадлежащая разности $H(\mathfrak{N}) \backslash \mathfrak{N} \mathfrak{\mathcal { A }}$. Группа $G$ разрешима по лемме 4, и для каждой ее нормальной подгруппы $N \neq 1$, в силу леммы 1 , верно, что $G / N \in H(\mathfrak{N})$. По индукции, $G / N \in \mathfrak{N} \mathfrak{U}$. Так как $\mathfrak{N} \mathfrak{U}-$ насыщенная формация, то группа $G$ примитивна ([4], с. 143). По теореме 4.42 в [4],

$$
F=F(G)=C_{G}(F) \simeq E_{p^{n}}
$$

- минимальная нормальная в $G$ подгруппа, а по уже доказанному утвеждению 3 теоремы $F-$ силовская подгруппа группы $G$.

Если $n=1$, то $G / F$ абелева как группа автоморфизмов группы $F$ простого порядка $p$, и $G \in \mathfrak{N} \Re$. Пусть $n \geqslant 2$. Для каждого $q \in \pi=\pi(G / F)$ подгруппа $[F] G_{q}$ является подгруппой Шмидта по лемме 5 , поскольку $[F] G_{q}$ - холлова ненильпотентная подгруппа. По п. 2 леммы 2, число $q$ делит $p^{n}-1$ и не делит $p^{n_{1}}-1$ для всех $1 \leqslant n_{1}<n$. Группа $G / F$ изоморфна подгруппе $G L(n, p)$, в которой имеется циклическая $\pi$-холлова подгруппа $T$ по лемме 6. По теореме 5.3.2 в [7], $G / F$ содержится в некоторой подгруппе $T^{x}, x \in G L(n, p)$. Поэтому $G / F$ циклическая, и $G \in \mathfrak{N} \mathfrak{A}$.

Теперь проверим, что $Z(G / Z(G))=1$. Допустим противное, и пусть $U / Z(G)$ - подгруппа простого порядка $t$ из $Z(G / Z(G))$, а $T$ - силовская $t$-подгруппа из $U$. Так как $U$ нильпотентна, то $T$ нормальна в $G$. Поскольку $T$ не содержится в $Z(G)$, то $T-$ силовская подгруппа группы $G$ по утверждению 3 теоремы. Так как $T Z(G) / Z(G) \simeq T / T \cap Z(G)$ циклическая, то, по лемме 1.56 в [4], подгруппа $T$ абелева. Если $G t$-нильпотентна, то $G t$-разложима и $T \subseteq Z(G)$, получаем противоречие. Значит, $G$ не $t$-нильпотентна, и существует $t$-замкнутая $t d$-подгруппа Шмидта $S$. Подгруппа $S Z(G) / Z(G)$ является $t d$-подгруппой, а по лемме $2 S Z(G) / Z(G)$ будет подгруппой Шмидта в $G / Z(G)$. Это противоречит тому, что $T Z(G) / Z(G) \subseteq Z(G / Z(G))$. Поэтому допущение неверно, и $Z(G / Z(G))=1$.

Пусть $G \in H(\Re)$ и $Z(G)=1$. Из уже доказанного утверждения 3 теоремы следует, что каждая минимальная нормальная в $G$ подгруппа является силовской в $G$ подгруппой. Поэтому $F(G)=A$ - абелева холлова подгруппа, у которой каждая силовская подгруппа минимальная нормальная в $G$ подгруппа. Пусть $B-$ дополнение к $A$ в $G$, оно существует по теореме Шура-Цассензауза (см. [4], с. 136). Из утверждения 1 теоремы следует, что порядок $B$ свободен от квадратов. Поскольку $G \in \mathfrak{N} \mathfrak{A}$, то $B$ абелева, поэтому она циклическая. Теорема доказана.

Доказательство следствия 1. Если центр группы единичен, то следствие справедливо ввиду утверждения 4 теоремы. Пусть $Z(G) \neq 1$ и $N$ - подгруппа простого порядка $p$ из $Z(G)$. К фактор-группе $G / N$ применима индукция, поэтому

$$
\bar{G}=[A / N](B / N),
$$

где $A / N$ - нильпотентная холлова подгруппа, а $B / N$ - циклическая подгруппа. Поскольку $N \subseteq Z(G)$, то подгруппы $A$ и $B$ нильпотентны, (см. [4], лемма 3.15). Если $A$ холлова, то по теореме Шура-Цассензауза $B=N \times B_{1}$ и $G=[A] B_{1}$ является полупрямым произведением нильпотентной холловой подгруппы $A$ и циклической подгруппы $B_{1}$, что и требуется доказать. Пусть $A-$ не холлова подгруппа, тогда $A=N \times A_{1}$, где $A_{1}-$ нильпотентная нормальная в $G$ холлова подгруппа, и $G=\left[A_{1}\right] B$. Пусть $B_{1}-$ произведение 
всех нормальных в $G$ силовских подгрупп из $B$, а $B_{2}$ - произведение всех ненормальных в $G$ силовских подгрупп из $B$. Ясно, что $G=\left[A \times B_{1}\right] B_{2}, A \times B_{1}$ - нормальная в $G$ холлова подгруппа, а все силовские подгруппы в $B_{2}$ циклические по утв. 1 теоремы. Так как $B_{2}$ нильпотентна, то $B_{2}$ циклическая. Итак, группа $G$ содержит нильпотентную холлову подгруппу, фактор-группа по которой циклическая. Из утверждения 4 теоремы следует, что производная длина группы не выше 3.

\section{Список литературы}

1. Шмидт О. Ю., Группы, все подгруппы которых специальные. Математический сборник (1924) 31, 366-372.

2. Huppert B., Endliche Gruppen, I. Springer, Berlin, 1967.

3. Монахов В. С., Подгруппы Шмидта, их существование и некоторые приложения. В сб.: Tруды Украинского математического конгресса. Институт математики, Киев, 2002, с. 81-90.

4. Монахов В. С., Введение в теорию конечных групп и их классов. Вышэйшая школа, Минск, 2006.

5. Беркович Я. Г., Теорема о ненильпотентных разрешимых подгруппах конечной группы. В сб.: Конечные группы. Наука и техника, Минск, 1966, с. 24-39.

6. Huppert B., Blackburn N., Finite groups, II. Springer, Berlin, 1982.

7. Suzuki M., Group theory, II. Springer, New York, 1986.

Статья поступила 21.05.2011. 